\title{
Retrospective access to data: the ENGAGE consent experience
}

\author{
Anne Marie Tassé ${ }^{\star, 1}$, Isabelle Budin-Ljøsne ${ }^{2}$, Bartha Maria Knoppers ${ }^{3}$ and Jennifer R Harris ${ }^{2}$
}

The rapid emergence of large-scale genetic databases raises issues at the nexus of medical law and ethics, as well as the need, at both national and international levels, for an appropriate and effective framework for their governance. This is even more so for retrospective access to data for secondary uses, wherein the original consent did not foresee such use. The first part of this paper provides a brief historical overview of the ethical and legal frameworks governing consent issues in biobanking generally, before turning to the secondary use of retrospective data in epidemiological biobanks. Such use raises particularly complex issues when (1) the original consent provided is restricted; (2) the minor research subject reaches legal age; (3) the research subject dies; or (4) samples and data were obtained during medical care. Our analysis demonstrates the inconclusive, and even contradictory, nature of guidelines and confirms the current lack of compatible regulations. The second part of this paper uses the European Network for Genetic and Genomic Epidemiology (ENGAGE Consortium) as a case study to illustrate the challenges of research using previously collected data sets in Europe. Our study of 52 ENGAGE consent forms and information documents shows that a broad range of mechanisms were developed to enable secondary use of the data that are part of the ENGAGE Consortium.

European Journal of Human Genetics (2010) 18, 741-745; doi:10.1038/ejhg.2010.30; published online 24 March 2010

\section{BIOBANK RESEARCH IN PERSPECTIVE}

The last decade has seen the rise of research consortia - the result of a convergence of needs, multidisciplinary collaboration, combined expertise, and larger cohorts. In parallel, the development of automated analyses of tissue collection and data ${ }^{1}$ has led to a rapid growth of both national and international and public and commercial biobanks. (In this article, the terms 'biobank', 'genetic database', 'biological repository' (...) are used interchangeably to signify any collection of human biological samples and/or data organized as a resource for genetic analysis and research, including material collected for research purposes, pathology collection, rare or specific disease archives, or any other databases created for longitudinal studies on any disease or condition.) Many ongoing longitudinal studies, often rich in health and sociodemographic information, have expanded their reach by adding biobank components.

Historically, North American and European regulatory approaches to biomedical research covered traditional clinical research and relatively small-scale biosample collections. Although research tools and resources have evolved, ethics and law lag far behind. ${ }^{2}$ This is even more so for retrospective access to data for secondary uses (secondary use is 'Using data or samples in a way that differs from the original purpose' ( $\mathrm{P}^{3} \mathrm{G}$ Observatory, Lexicon, online: < http://www.p3gobservatory.org/lexicon/list.htms > (21 May, 2009)), in which the original consent did not foresee such use.

The first part of this paper provides a brief historical overview of the ethical and legal frameworks governing consent issues in biobanking before 1995 (A) and then in the last decade (B). The second part of this paper uses the European Network for Genetic and Genomic Epidemiology (ENGAGE Consortium), ${ }^{3}$ a large European collaborative research project with 25 partners from 13 countries as a case study to illustrate the challenges of research using previously collected data sets in Europe. We describe the general characteristics of ENGAGE consents (A) before examining the possibility of applying the 'prospective' solutions found in modern biobanks for such a 'retrospective' research (B). The majority of studies comprising the ENGAGE Consortium were recruited on the basis of their ability to deliver already genome-scanned data to the ENGAGE analyses at the start of the project in January 2008. This particular sample of studies may represent European cohort studies that, at that time, were at the forefront of genomic research. Given that the data had been consented long before ENGAGE was conceived, our primary interest was to assess the consent mechanisms that permitted these data to be used in ENGAGE and the specific challenges encountered. Striving to generalize these results to other consortia is outside the scope of our focus and may not be very relevant because of the inherent idiosyncracies of the consortia, including variations in their research focus and the criteria used to recruit studies. Moreover, this is not an analysis of the national legal frameworks governing retrospective research.

\section{PART I. HISTORICAL, LEGAL, AND ETHICAL BIOBANKING ISSUES: INTERNATIONAL POLICY PERSPECTIVES}

The rapid growth of biobanks has been followed by a concomitant growth of international, national, and professional guidelines, as well as recommendations, laws, and regulations. ${ }^{4}$ Although the very creation of biobanks was questioned only a few years ago, today it is the issues they raise that predominate. Our focus is on the issues raised by consent to the secondary use of data. Section A studies the barriers created by informed consent requirements as found in international guidelines from 1947 to 1995, whereas section B examines the solutions developed since then.

${ }^{1}$ Université de Montréal, Faculté de droit, Montreal, Canada; ${ }^{2}$ The Norwegian Institute of Public Health, Oslo, Norway; ${ }^{3}$ McGill University, Centre of Genomics and Policy, Faculty of Medicine, Department of Human Genetics, Montreal, Canada

*Correspondence: AM Tassé, Université de Montréal, 740, Dr Penfield Ave., suite 5202, Montreal, Quebec, Canada, H3A 1A4. E-mail: anne-marie.tasse@umontreal.ca 
(A) Consent in international guidelines from 1947 to 1995:

\section{barriers?}

Consent, as it is conceived in international guidelines from 1947 to 1995, endorses positions that can create barriers to research and are now being revisited in light of advances in science and data use. These include (i) the requirement for individual informed consent, as well as (ii) access to samples and data collected from minors or (iii) from subjects who are now deceased.

(i) Requirement for informed consent. Respect for autonomy is one of the basic ethical principles of research ethics, dictating the requirement for a previous, free and informed consent for research participants. ${ }^{5}$ Most authors consider informed consent as the gold standard of research ethics. ${ }^{6}$ The classical doctrine of informed consent requires a participant to be appropriately informed about the research objectives, ${ }^{7-16}$ procedures, ${ }^{9,10,13,15,16}$ risks, ${ }^{9-11,13,15-17}$ and benefits. ${ }^{10,11,13,15-17}$ There are different information and consent procedures for different kinds of research. The use of biological samples and data outside the range of research mentioned in the consent form would be considered a 'secondary use', which, in most jurisdictions, is neither legal nor ethical in the absence of new consent, ethics waiver, or legal provisions.

(ii) Minors. The inclusion of children in longitudinal studies also raises ethical and legal issues, asi it is their parents who authorize such participation. ${ }^{10-14,16,18,19}$ As children mature and develop the capacity to make independent decisions, they have an increased capacity to participate in decision making. ${ }^{19}$ This raises questions about the scope of parental consent and the assent/consent of the child. International policies agree that informed consent of parents (or legal representatives) is required before collecting, storing, and using the data and biological samples of a child. ${ }^{10-14,16,18}$ As consent is a continuous process, what happens when the child requests control over his or her own participation in a research biobank and refutes a parental decision? This is particularly relevant for population biobanks and consortia in which samples and data are stored for long, sometimes undefined periods. Moreover, in some jurisdictions, the law recognizes the growing capacity of children. Mature minors then can make an independent choice regarding their continued participation.,19 Therefore, some doctrines propose to seek consent from the children when they reach sufficient maturity but others limit participation without parental authorization to legal age. ${ }^{20}$ This could be difficult for biobanks, unless researchers follow up with the parent(s) and child on a regular basis. ${ }^{21}$

(iii) Deceased individuals. The legal and ethical requirements of obtaining consent for each new research project also create hurdles when research subjects are deceased. The secondary use of research samples and data collected for a specific research project after the subjects' death is rarely discussed in international guidelines. Although some guidelines recognize that access to genetic data after the death of the person from whom it was obtained presents special issues, ${ }^{12}$ other guidelines recommend that biobanks should have a clearly articulated policy about the effects, if any, of the participant's death or loss of legal capacity, and that participants should be informed of this policy. ${ }^{15}$ Although this solution might be valuable for prospective cohorts, it would be impossible to implement for retrospective population biobanks, as consents have already been collected.

\section{(B) Consent in international guidelines 1995: solutions?}

Since 1995, guidelines began to recognize the barriers to population and consortia research. Proposed solutions include the following: (i) broad/blanket consent; (ii) multilayered consent with secondary use statements; (iii) recontact/reconsent mechanisms; (iv) presumed consent/opting out; (v) waived consent; and (vi) anonymized $v s$ coded data.

(i) Broad/blanket consent. Since 1998, some guidelines propose the use of a broad or even a blanket consent ${ }^{12,22,23}$ 'that would allow use of a sample for genetic research in general, including future as yet unspecified projects. ${ }^{22}$ Thus, only if a research project is different from the wide ambit of the original broad/blanket consent would a new consent be necessary. ${ }^{4}$ However, such consent can only be implemented prospectively. If research is retrospective, recontacting subjects to obtain a new informed consent is required by most international guidelines. ${ }^{13,15,16,24}$

(ii) Multilayered consent with secondary use. A North American solution to overcome the hurdles created by the requirement for a specific informed consent was a 'multilayered consent'. This solution, proposed a decade ago, ${ }^{25,26}$ suggests dealing with the secondary use of genetic material and associated data by using a comprehensive consent form, which allows the research subject to choose from a number of options in advance. Adding a 'secondary use statement' that mentions that even if samples and data are collected for a defined research or for research in a defined domain they can be used for other research in different domains is an example of this 'anticipatory' practice. Respecting multilayered consent, however, requires impressive and expensive logistics. In the context of population biobanks and international consortia, it is impracticable and impossible.

(iii) Presumed consent with opting out. The requirement for informed consent for research participants has always had as its counterpart the right to withdraw. ${ }^{9-15}$ Under a presumed consent approach with notification, the counterpart is the possibility to opt out. ${ }^{4,27}$ Presumed consent with an 'opt-out' mechanism requires a wide notification of the public and the creation of a structure to ensure the respect of those who opt out. This structure already exists in some European countries, with regard to the secondary use of clinically obtained samples and data for research purposes. Indeed, France, ${ }^{28,29}$ the Netherlands, ${ }^{30}$ and Spain $^{31}$ foresee this notification with an opt-out mechanism for the research use of leftover tissues in the setting of medical care.

(iv) Recontact/reconsent. For population biobanks with longitudinal objectives, to continually recontact participants for new consents defeats the purpose, that is, the creation of a longitudinal resource for future unspecified research. It can also be impossible to obtain a new consent from research subjects who have died or who have moved. ${ }^{8}$ Some subjects can also resent the recontact as being offensive or an invasion of their privacy. ${ }^{8}$ For these reasons, population studies now ask, at the time of recruitment, for permission to recontact participants if they have additional questions or need for samples. ${ }^{8}$

(v) Waived consent. If it is not possible to recontact participants for reconsent, some guidelines allow for waived consent for the use of biological material, if certain conditions are met. ${ }^{4}$ However, these conditions are not harmonized among international guidelines. $^{10,13,16-18,24}$

(vi) Anonymized vs coded data. As stated in the Declaration of Helsinki, ethical principles apply to 'medical research involving human subjects, including research on identifiable human material or identifiable data. ${ }^{10}$ It follows that research using anonymized or anonymous data does not create an obligation to obtain informed consent, as the study does not involve identifiable individuals. ${ }^{10,24}$ 
However, the use of anonymized data is not possible in all research and creates its own ethical hurdles, as some researchers need to follow up participants over time, research subjects cannot withdraw or be recontacted for reconsent, and it is impossible to repeat the analyses for validation purposes. Moreover, anonymization may not always be appropriate. In the case of research focusing on gene-environment interactions, personalized and sometimes even identifiable health data may be required for a complete analysis. For these reasons, some countries such as the Netherlands and United States have developed a data coding system allowing for the secondary use without reconsent of coded data as if it were anonymized, as the researcher accessing data does not have the link back to the participant. ${ }^{32}$

The barriers and solutions described in this section might seem purely theoretical, but they have important implications for research, especially for retrospective meta-analyses such as those conducted in the ENGAGE project.

\section{PART II. LEGAL AND ETHICAL ISSUES OF BIOBANKING: THE} CASE OF THE ENGAGE CONSORTIUM

In this second part, we use the ENGAGE Consortium as a case study to examine the impact of the challenges of research using previously collected data sets. First, we describe the general characteristics of the studies comprising ENGAGE and the nature of their original consents (A). Next, we analyze the information given to the research participants and how these documents deal with issues identified as potential hurdles for retrospective research (B).

\section{(A) General characteristics: ENGAGE}

The 39 ENGAGE cohorts originate from 13 different countries and are part of population-based follow-up studies (19), family studies (7), twin studies, (6) and case-control settings or case-only studies (7)..$^{33}$ The size of the ENGAGE cohorts varies from data sets of $<10000$ samples per data set ( $67 \%$ of all cohorts) to data sets of $>50000$ samples per data set ( $6 \%$ of all cohorts). Collectively, the data from these 39 cohorts represent human genetics data sets from $>600000$ individuals. ENGAGE is a 5-year project that was funded by The European Community's Seventh Framework Programme (FP7/20072013) in 2008 .

The genetic and phenotypic data made available to ENGAGE were collected at a time when their possible future use in ENGAGE was unknown. Analyses of electronic copies of the information leaflets and informed consent forms ('Information Documents' or ID) used in the ENGAGE cohort studies identified the main challenges emerging from the use of already collected data for the purpose of retrospective research. In all, 52 studies from the 39 cohorts of ENGAGE were analyzed. This represents $95 \%$ of the ENGAGE cohort studies. Although six ENGAGE partners are from Nordic countries, $62 \%$ (32) of the cohort studies are from Nordic countries. It should be noted that the results below are based on our analysis of the content of the information documents given to the participants. Neither other consent mechanisms used in different countries nor national legal frameworks were investigated.

About two-thirds of the ENGAGE cohort studies are regionally representative (34), with participants recruited from specific, small, or medium-size towns or regions, usually through the county hospital or the regional biobank. The remaining studies (18) recruited participants countrywide. Furthermore, $88 \%$ (46) of the data collected in the 52 cohort studies are $<20$ years old. Most of the studies are longitudinal designs and were often added onto similar previous studies (eg, KORA, Tromsø, NFBC). The majority recruited only adults; $13 \%$ (7) also recruited minors, usually for the purpose of following up mothers and their infants until a certain age.

All studies had local research ethics committee approval, and research participants received an information sheet describing the research and/or an informed consent form at the time of recruitment. Normally, clinical and health information was collected by means of questionnaires and samples (eg, blood sample, urine sample). Some studies included more in-depth medical examinations (eg, ECG).

\section{(B) Mechanisms within ENGAGE}

The information documents given to the research participants describe the mechanisms used in retrospective research under the following categories: (i) broad consent; (ii) multilayered consent; (iii) secondary use; (iv) withdrawal; (v) recontact/reconsent; (vi) deceased persons; and (vii) anonymized/coded data. These will be briefly described before analyzing their impact on ENGAGE (viii). Figure 1 shows the frequency at which these various mechanisms were used by ENGAGE cohorts.

(i) Broad consent. Consents can be categorized into two main types. Broad consent is defined as asking the research participants to consent to a wide range of future research, ${ }^{34}$ whereas specific consent is limited to a specific research. In all, $52 \%$ (27) of the studies used broad consent and $48 \%$ (25) used specific consent. In 67\% (35) of the studies, the information documents specified that the samples taken would be used for DNA extraction or genetic analysis. In the remaining $33 \%$ (17), the information provided to participants did not mention genetic research per se but only biological research (eg, extraction of clinical values such as cholesterol levels).

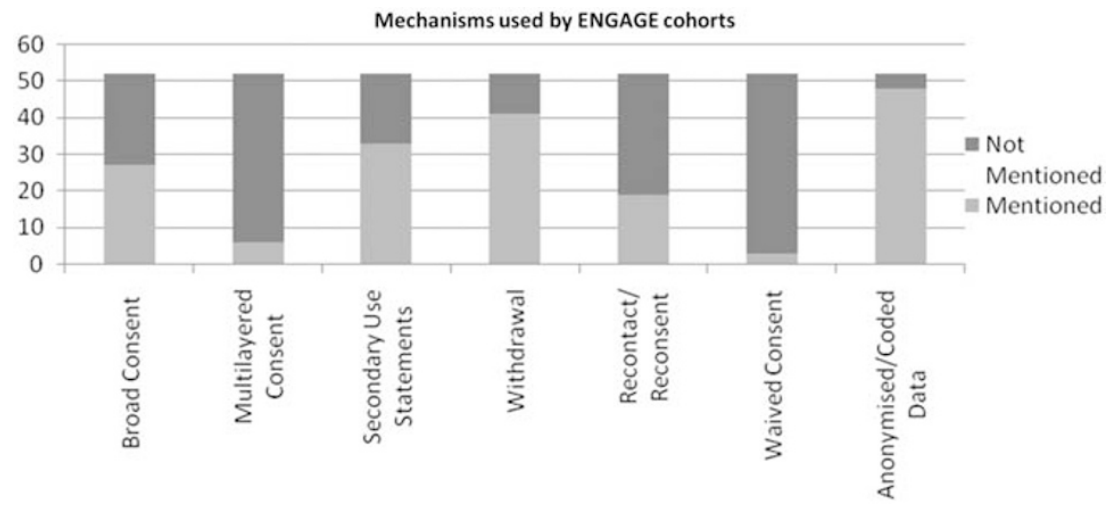

Figure 1 Mechanisms used by ENGAGE cohorts. Source: informed consent forms and information leaflets from 52 studies belonging to the 39 ENGAGE cohorts. 
(ii) Multilayered consent. In all, $12 \%$ of studies (6) used a multilayered consent approach. For instance, in some informed consent forms from Norway and Sweden, research participants were given the option to cross out items they did not want to give their consent to (eg, extraction of DNA, recontact for further investigation, merging of results with other registers).

(iii) Secondary use. A total of $63 \%$ (33) of the studies mentioned secondary use of collected data. In those 33 studies, seven specified that such research would only be carried out with the approval of a local oversight authority, such as a data inspectorate and/or a research ethics committee. Of the studies, 54\% (28) mentioned that the data may be shared with other biobanks or research institutions. In one of three studies (36\%), secondary use is not mentioned in the information document. Failure to mention possible secondary use does not necessarily preclude such use, as the national ethical-legal frameworks may not explicitly prohibit it.

(iv) Withdrawal. In $79 \%$ of the studies (41), participants were informed about their right to withdraw from the study. However, the fate of the data and samples on withdrawal is not dealt with uniformly. They can be destroyed, removed, or kept in the biobank. In about one-third of the studies, in which withdrawal is mentioned, the procedure to be followed is not specified.

(v) Recontact/reconsent. Of the studies, 36\% (19) mention that participants may be recontacted if new research is to be performed or if the data are to be shared with other investigators or biobanks. Four studies mention explicitly that such recontact implies that a new consent will be obtained. In 13\% (7) of the studies, mothers and infants were recruited, usually for longitudinal studies with follow-up until a certain age. Among these, two address the issue of informing minors of their participation in the study or obtaining new consent once they have matured or reached legal age. In the first study, researchers would inform the minor of his/her participation at the age of 15 and 18 years. In the second study, researchers inform the minor of data storage and of the possibility of withdrawal.

(vi) Deceased persons. Only 5\% (3) of studies explicitly waive consent for the use of data and samples after the death or incapacity of the research participant. In the remaining 49 studies, this issue is not addressed.

(vii) Anonymized/coded data. A total of 92\% (48) of the information documents describe respect for confidentiality and the secure use of the health information of participants. In most cases, it is mentioned that the data will be deidentified (eg, coded) or that the participant's identity will not be traceable (anonymized).

(viii) Impact for ENGAGE. In all, 73\% of ENGAGE cohort studies use at least one mechanism to facilitate the secondary use of data. Ten studies (19\%) use four mechanisms, whereas six use three mechanisms (Figure 2). Four cohort studies use broad consent in conjunction with another mechanism, either a secondary use statement or a recontact/ reconsent mechanism, whereas seven (13\%) studies use only a broad consent. Even among the $11(21 \%)$ studies that have a specific consent, 25 cohorts foresee the use of different mechanisms, such as a secondary use statement (7), a recontact/reconsent mechanism (2), a combination of a secondary use statement with a waiver of consent (1), or a recontact/reconsent mechanism with a waiver of consent (1).

Only 14 studies (27\%) have no explicit mechanism for the secondary retrospective use of data. Therefore, the data collected by these cohorts cannot be used by ENGAGE partners, unless in accordance with the original consent, or because national legislative and/or ethical frameworks allow such secondary use without the reconsent of the research participant or under an ethics waiver.

It is interesting to note that for the main research areas of ENGAGE (cardiovascular diseases and/or diabetes), 85\% (44) of the studies obtained consent to perform such research. This consent corresponds to the overall research objectives of ENGAGE for the majority of research participants of the 52 cohort studies analyzed.

Nevertheless, the practical management of change of status (death, incapacity, reaching legal age, or withdrawal from the study), the secure exchange of data across institutions and borders, and the risk for reidentification represent important challenges for ENGAGE. Because national legislation differs from one country to another, managing data exchange in ENGAGE requires knowledge of existing regulations and a continuous follow-up of international norms. When entering the project, all partners were responsible for ensuring that their data would be used in accordance with the consent given by the research participants. Difficulties may still occur when seeking a renewed consent or approval from a local research ethics committee or data inspectorate. This could lead to expensive and burdensome administrative procedures for the ENGAGE project. These challenges are not addressed in the consent forms. The project, which has already produced a significant number of international scientific publications (the list of ENGAGE publications is available on the ENGAGE public web site www.euengage.org.), illustrates the need for the evolution of consent, offers a unique opportunity to further work on these issues, and, at the same time, continue to carry out major scientific research.
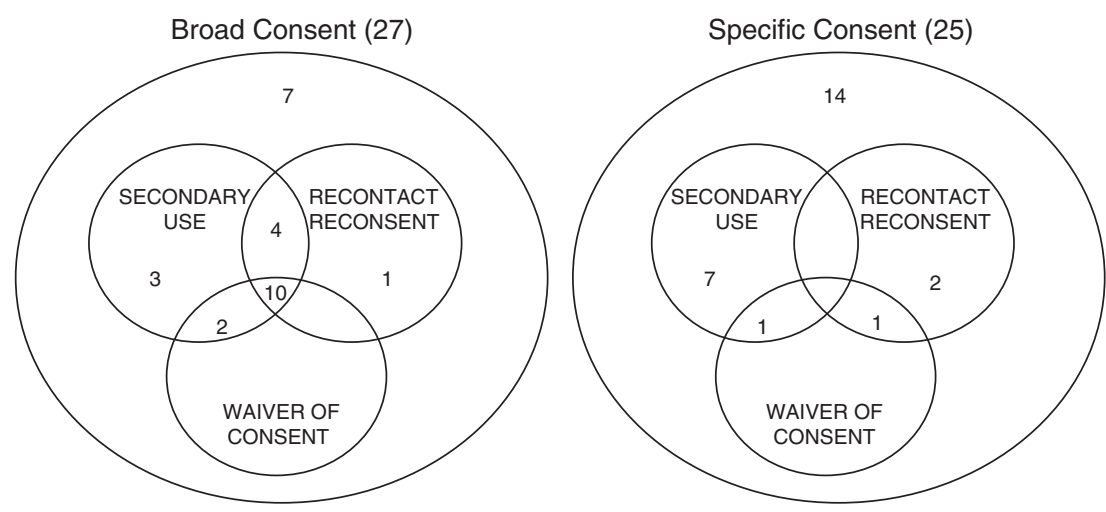

Figure 2 Solutions among ENGAGE cohort studies. 


\section{CONCLUSION: NEED FOR COHERENT AND COMPREHENSIVE ANSWERS}

Current legal and ethical frameworks governing research are largely concerned with clinical trials, and therefore represent an 'individualoriented' approach. ${ }^{35}$ Many of the ethical and policy issues raised by biobanks revolve around tensions between individual 'rights', emerging from legal documents and ethical guidelines, and the objectives of biobanks. ${ }^{7}$ We have demonstrated that the secondary use of retrospective data in epidemiological biobank research raises particularly complex issues if the consents are inconclusive, contradictory, or incompatible with such research.

The ENGAGE partners developed a broad range of mechanisms to enable secondary use of data. This particular sample of studies may represent European cohort studies that, at that time, were at the forefront of genomic research. Striving to generalize these results to other consortia is outside the scope of our focus and may not be very relevant because of inherent scientific differences between consortia.

The study of barriers hampering secondary use of data within the ENGAGE Consortium shows an urgent need for a harmonized and 'user friendly' international ethico-legal framework for governing biobanks ${ }^{36}$ that foresee 'going back' as well as going forward. Retrospective research also needs a harmonized governance framework such as that developed for prospective research (see UK Ethics and Governance Framework ${ }^{37}$ ). However, scientific complexity, together with a multiplicity of international and national factors, could impede such an initiative. Another solution could be the creation of tools or of a platform with a common international ethical framework for retrospective research. Those who participated in past research deserve to have their contribution used and recognized.

\section{CONFLICT OF INTEREST}

The authors declare no conflict of interest.

\section{ACKNOWLEDGEMENTS}

We thank Dr Helena Hääriäinen, Secretary General, European Society of Human Genetics, National Public Health Institute, Finland, for the translation of Finnish documents, and Dr Irina Costea, Knowledge Translation Officer, Public Health Agency of Canada, Canada, for the translation of German documents. This research was supported through funds from The European Community's Seventh Framework Programme (FP7/2007-2013), ENGAGE Consortium, grant agreement HEALTH-F4-2007-201413

1 Biller-Andorno N, Boggio A, Elger B, Ganguli-Mitra A, Capron A, Mauron A: Ethical issues regarding research biobanks: aims, methods, and main results of a qualitative study among international and US experts; in Elger B, Biller-Andorno N, Mauron A, Capron A (dir.) (eds): Ethical Issues in Governing Biobanks: Global Perspectives. Hampshire: Ashgate Publishing Limited, 2008, pp 39-56.

2 Knoppers BM: Genomics and policymaking: from static models to complex systems? Hum Genet 2009; 125: 375-379.

3 European Network for Genetic and Genomic Epidemiology (ENGAGE). Available from: http://www.euengage.org/.

4 Vayena E, Ganguli-Mitra A, Biller-Andorno N: Guidelines on biobanks: emerging consensus and unresolved controversies; in Elger B, Biller-Andorno N, Mauron A, Capron A (dir.) (eds): Ethical Issues in Governing Biobanks: Global Perspectives. Hampshire: Ashgate Publishing Limited, 2008, pp 23-35.

5 Ries NM: Growing up as a research subject: ethical and legal issues in birth cohort studies involving genetic research. Health Law J 2007; 15: 1-41.

6 Elger B: Consent and use of samples; in Elger B, Biller-Andorno N, Mauron A, Capron A (dir.) (eds): Ethical Issues in Governing Biobanks: Global Perspectives. Hampshire: Ashgate Publishing Limited, 2008, pp 57-88.

7 Mauron A: Introduction: biobanks, genomics, and research - a nightmare for public policy makers?; in Elger B, Biller-Andorno N, Mauron A, Capron A (dir.) (eds): Ethical
Issues in Governing Biobanks: Global Perspectives. Hampshire: Ashgate Publishing Limited, 2008, pp 1-9.

8 Knoppers BM, Abdul-Rahman $\mathrm{MH}$ : Biobanks in the literature; in Elger B, BillerAndorno N, Mauron A, Capron A (dir.) (eds): Ethical Issues in Governing Biobanks: Global Perspectives. Hampshire: Ashgate Publishing Limited, 2008, pp 13-22.

9 Trials of War Criminals before the Nuremberg Military Tribunals under Control Council Law: Nuremberg Code. Nuremberg, October 1946: US Government Printing Office 1949, No 10, pp 181-182.

10 World Medical Association (WMA): World Medical Association Declaration of Helsinki: Ethical Principles for Medical Research Involving Human Subjects. Adopted by the 18th WMA General Assembly, Helsinki, Finland, June 1964, and amended in 1975, 1983, 1989, 1996, 2000, 2002, 2004 and 2008.

11 Council of Europe (COE): Convention for the Protection of Human Rights and Dignity of the Human Being with regard to the Application of Biology and Medicine: Convention on Human Rights and Biomedicine. Oviedo: Council of Europe, 1997.

12 United Nations Educational, Scientific and Cultural Organisation (UNESCO): Human Genetic Data: Preliminary Study by the IBC on its Collection, Processing Storage and Use. Paris: United Nations Educational, Scientific and Cultural Organisation, 2002.

13 Council for International Organizations of Medical Sciences (CIOMS), World Health Organization (WHO): International Ethical Guidelines for Biomedical Research Involving Human Subjects. Geneva: CIOMS Publications, 2002.

14 United Nations Educational, Scientific and Cultural Organisation (UNESCO), International Bioethics Committee (IBC): International Declaration on Human Genetic Data. Paris: United Nations Educational, Scientific and Cultural Organisation, International Bioethics Committee, 2003.

15 Organisation for Economic Co-operation and Development (OECD): Draft Guidelines for Human Biobanks and Genetic Research Databases: Text for Comment. Paris: Organisation for Economic Co-operation and Development, 2008.

16 Council for International Organizations of Medical Sciences (CIOMS): International Ethical Guidelines for Epidemiological Studies. Geneva: CIOMS Publications, 2009.

17 Human Genome Organisation (HUGO): Statement on the Principled Conduct of Genetics Research. London: Eubios Journal of Asian and International Bioethics, 1995; 6: 59-60.

18 United Nations Educational, Scientific and Cultural Organisation (UNESCO): Universal Declaration on the Human Genome and Human Rights. Paris: United Nations Educational, Scientific and Cultural Organisation, 1997.

19 Samuël J, Ries NM, Malkin D, Knoppers BM: Biobanks and longitudinal studies: where are the children? GenEdit 2008; 6: 1-8.

20 Burke W, Diekema DS: Ethical issues arising from the participation of children in genetic research. J Pediatr 2006; 149: S34-S38.

21 Gurwitz D, Fortier I, Lunshof JE, Knoppers BM: Children and population biobanks. Science 2009; 325: 818-819.

22 World Health Organisation (WHO): Proposed International Guidelines on Ethical Issues in Medical Genetics and Genetic Services. Geneva: World Health Organisation, 1998.

23 Human Genome Organisation (HUGO) Ethics Committee: Statement on Human Genomic Databases. London: Eubios Journal of Asian and International Bioethics, 2003; 13: 99.

24 World Medical Association (WMA): The World Medical Association Declaration on Ethical Considerations Regarding Health Databases. Washington: World Medical Association, 2002.

25 National Bioethics Advisory Commission (NBAC): Research Involving Human Biological Materials: Ethical Issues and Policy Guidance, Volume I: Report and Recommendations. Rockville: National Bioethics Advisory Commission, 1999.

26 Canadian Institutes of Health Research (ClHR), Natural Sciences and Engineering Research Council of Canada (NSERC), Social Sciences and Humanities Research Council of Canada (SSHRC): Tri-Council Policy Statement: Ethical Conduct for Research Involving Humans. Ottawa: The Interagency Advisory Panel on Research Ethics (PRE), 1998 (with 2000, 2002, 2005 amendments).

27 Human Genome Organisation (HUGO) Ethics Committee: Statement on DNA Sampling: Control and Access. London: Human Genome Organisation, 1998.

28 French Republic: Loi $n^{\circ} 78-17$ du 6 janvier 1978 relative à l'informatique, aux fichiers et aux libertés. Paris: J.O., consolidated version 14 May 2009.

29 French Republic: Code de la santé publique. Paris: J.O., consolidated version August 29th 2009, s. L1211-2.

30 Dutch government: Act of 17 November 1994 amending the Civil Code and other legislation in connection with the incorporation of provisions concerning contract to provide medical treatment. The Hague, s. 458.

31 Spain Parliament: Law 14/2007, of 3 July, on Biomedical Research. Madrid, s.58(2).

32 Office for Human Research Protections (OHRP): Guidance on Research Involving Coded Private Information or Biological Specimens. Washington: Office for Human Research Protections, 2008.

33 ENGAGE. Grant Agreement no 201413, Annex 1, version 3-2.

34 Hofmann B: Broadening consent - and diluting ethics? J Med Ethics 2009; 35: 125-129.

35 Dumez B, Van Damme K, Casteleyn L: Research on ethics in two large Human Biomonitoring projects ECNIS and NewGeneris: a bottom up approach. Environ Health 2008; 7: S1-S7.

36 Harris JH, Burton P, Brand A et al: Report and Recommendations: Networking Meeting for EU-Funded Biobanking Projects. Brussels, 2008.

37 UK Biobank: UK Biobank Ethics and Governance Framework. Adswood, 2007. 